\title{
The C/EBPß-Dependent Induction of TFDP2 Facilitates Porcine Reproductive and Respiratory Syndrome Virus Proliferation
}

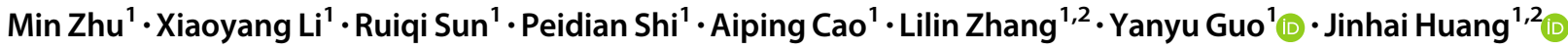

Received: 7 February 2021 / Accepted: 28 April 2021 / Published online: 17 June 2021

(c) Wuhan Institute of Virology, CAS 2021

\begin{abstract}
Porcine reproductive and respiratory syndrome (PRRS) is an important infectious disease caused by porcine reproductive and respiratory syndrome virus (PRRSV), leading to significant economic losses in swine industry worldwide. Although several studies have shown that PRRSV can affect the cell cycle of infected cells, it is still unclear how it manipulates the cell cycle to facilitate its proliferation. In this study, we analyzed the mRNA expression profiles of transcription factors in PRRSV-infected 3D4/21 cells by RNA-sequencing. The result shows that the expression of transcription factor DP2 (TFDP2) is remarkably upregulated in PRRSV-infected cells. Further studies show that TFDP2 contributes to PRRSV proliferation and the PRRSV nucleocapsid $(\mathrm{N})$ protein induces TFDP2 expression by activating C/EBP $\beta$. TFDP2 positively regulates cyclin A expression and triggers a less proportion of cells in the S phase, which contributes to PRRSV proliferation. This study proposes a novel mechanism by which PRRSV utilizes host protein to regulate the cell cycle to favor its infection. Findings from this study will help us for a better understanding of PRRSV pathogenesis.
\end{abstract}

Keywords Porcine reproductive and respiratory syndrome virus (PRRSV) - Transcription factor DP2 (TFDP2) · Cell cycle $\cdot$ Cyclin A

\section{Introduction}

Porcine reproductive and respiratory syndrome (PRRS), first occurred in the United States in 1987, is an endemic disease causing huge economic losses to pig farms around the world (Garner et al. 2001; Neumann et al. 2005; Rossow 1998). PRRSV, the causative agent of PRRS, belongs to the family Arteriviridae in the order

Supplementary Information The online version contains supplementary material available at https://doi.org/10.1007/s12250021-00403-w.

Min Zhu and Xiaoyang Li have contributed equally to this work.

Jinhai Huang

jinhaih@tju.edu.cn

$\triangle$ Yanyu Guo

146182@tju.edu.cn

1 School of Life Sciences, Tianjin University, Tianjin 300072, China

2 Tianjin Key Laboratory of Function and Application of Biological Macromolecular Structures, Tianjin University, Tianjin 300072, China
Nidovirales (Cavanagh 1997). It exists as two distinct virus species, that is, PRRSV-1 and PRRSV-2 (Adams et al. 2017; Wang et al. 2020). The PRRSV genome is a single-stranded positive-sense RNA, about $15 \mathrm{~kb}$ in length with 10 open reading frames (ORF) encoding eight structural proteins and at least 14 non-structural proteins (nsps) (Chand et al. 2012; Dokland 2010; Meulenberg 2000; Montaner-Tarbes et al. 2019). The clinical outcomes of PRRSV infection are characterized by severe reproductive failure in pregnant sows, pneumonia in piglets, and increased mortality of pigs at all ages (Music and Gagnon 2010).

Transcription factor DP2 (TFDP2), first isolated in 1995, forms heterodimers with the E2F family of transcription factors (Rogers et al. 1996; Wu et al. 1995). The binding of DP2 to E2F potentiates the DNA affinity and transcriptional activity of E2F (Wu et al. 1995; Zhang and Chellappan 1995). The target genes of E2F/DP heterodimers are implicated in a variety of cellular processes including cell cycle progression, DNA repair, cell differentiation, and apoptosis (Hitchens and Robbins 2003; Lam and La Thangue 1994). The eukaryotic cell cycle can be divided into four stages: G1, S, G2 and mitosis (M) (Wenzel and Singh 2018). It has been 
reported that some viruses can affect host cell cycle progression to provide a cellular environment that is advantageous for viral proliferation (Balistreri et al. 2016; Grey et al. 2010; Laichalk and Thorley-Lawson 2005; Swanton and Jones 2001). The cell cycle progression is tightly regulated by the binding of cyclins and cyclin-dependent kinases (CDKs) (Morgan 1995). It has been shown that PRRSV can arrest the cell cycle of MARC-145 cells (Song et al. 2018). However, how PRRSV manipulates the cell cycle to facilitate its proliferation is still unclear.

In this study, the mRNA expression profiles of transcription factors in PRRSV-infected porcine alveolar macrophage 3D4/21 cells were analyzed. The transcription factor TFDP2 was found to increase in PRRSV-infected macrophages, and in turn, it facilitated the PRRSV proliferation. The relationship between TFDP2 and PRRSV proliferation was further investigated. Our study provides new ideas for the prevention and control of PRRSV.

\section{Materials and Methods}

\section{Cells, Virus, and Antibody}

Porcine alveolar macrophage (PAM) cell lines CRL2843CD163 (3D4/21) were kindly provided by Prof. Jun Han from China Agricultural University and maintained in RPMI-1640 medium (Gibco, Carlsbad, CA, USA) supplemented with $10 \%(\mathrm{~V} / \mathrm{V})$ fetal bovine serum (FBS, Biological Industries, Kibbutz Beit-Haemek, Israel) and 100 $\mathrm{U} / \mathrm{mL}$ penicillin, and $100 \mu \mathrm{g} / \mathrm{mL}$ streptomycin. Human embryonic kidney (HEK) $293 \mathrm{~T}$ cells were cultured in Dulbecco's modified Eagle's medium (DMEM, Gibco) supplemented with $10 \%$ FBS. All cells were maintained at $37{ }^{\circ} \mathrm{C}$ with 5\% $\mathrm{CO}_{2}$. HP-PPRSV JXwn06 was used in this work and the titer was identified to be $10^{4} \mathrm{PFU} / \mathrm{mL}$ as previously described (Su et al. 2018).

Anti-PRRSV Nsp2 monoclonal antibody was kindly contributed by Prof. Jun Han from China Agricultural University. A polyclonal antibody against TFDP2 was generated in our laboratory. anti-FLAG antibody and anti-p-C/ EBP $\beta$ antibody were purchased from Cell Signaling Technology (CST, Boston, MA, USA). Anti-C/EBP $\beta$ antibody was purchased from ImmunoWay Biotechnology (Plano, TX, USA). Anti- $\beta$-actin antibody and secondary antibodies were purchased from Invitrogen (Carlsbad, CA, USA).

\section{RNA-Sequencing}

3D4/21 cells were seeded in 6-well plates and mockinfected or infected with 0.5 MOI PRRSV for $24 \mathrm{~h}$. Then cells were washed twice by PBS and added $1 \mathrm{~mL}$ Trizol reagent (Invitrogen, Carlsbad, CA, USA). The treated cells were sent to the Guangzhou GENE DENOVO Company for RNA sequencing. Data and a heatmap of differentially expressed genes were analyzed by Heml software.

\section{Plasmid Construction}

The gene encoding TFDP2 (GenBank Accession No. XM_021069556.1) was amplified from 3D4/21 cells cDNA using the primers listed in Supplementary Table S1 and cloned into pGEM ${ }^{\circledR}-\mathrm{T}$ Easy Vector (Transgen, Beijing, China). The CDS region of TFDP2 was amplified by PCR using specific primer pairs (Supplementary Table S1) and subcloned into the pFlag-CMV2 vector using a one-step clone kit (Vazyme, Nanjing, China).

Genomic DNA was extracted from PAMs using a DNA extraction kit (TaKaRa, Otsu, Shiga, Japan). The 1701-bp porcine TFDP2 promoter sequence (NC_010455.5) relative to the transcription initiation site $(+1)$ was amplified by specific primers and ligated into luciferase reporter vector pGL3-Basic (named - 1301/400-Luc) using a one-step clone kit (Vazyme, Nanjing, China). The truncated mutants of TFDP2 promoter were then constructed by PCR using the $-1301 / 400$-Luc plasmid as a template $(-792 / 400$ Luc, - 67/400-Luc, - 17/400-Luc). Site-directed mutagenesis of the C/EBP- $\beta$, ATF-1, AP-1, SP1 binding sites was performed by PCR using the $-17 / 400$-Luc vector as a template. The 1291-bp porcine cyclin A promoter (NC_010450.4) luciferase reporter plasmid was constructed as above described. The pRL-TK Renilla luciferase reporter plasmid was used as an internal control. All primers are listed in Supplementary Table S1.

\section{Quantitative Real-Time Reverse-Transcription PCR (real-time RT-qPCR)}

Total RNA was extracted from 3D4/21 cells using TRIzol reagent (Invitrogen, Carlsbad, CA, USA) and the first-strand cDNA was synthesized using a First-Strand Synthesis System (Transgen, Beijing, China) according to the manufacturer's instructions. The relative gene expression was analyzed by real-time RT-qPCR carried out on qTOWER $^{3} \mathrm{G}$ Real-time PCR system (Analytik Jena AG, Jena, Germany). Relative gene expression levels were calculated using the comparative cycle threshold (CT) method according to the manufacturer's protocol (Applied Biosystem, Foster City, CA, USA). All data presented are relatively quantitative, normalized to the $\beta$-actin and analyzed using GraphPad Prism 8.0 software. All primers used for real-time RT-qPCR are listed in Supplementary Table S2. 


\section{Western Blot Analysis}

Cells were lysed in RIPA buffer (Solarbio, Beijing, China) with $100 \mathrm{U}$ of proteinase inhibitors phenylmethanesulfonyl fluoride [PMSF] (Solarbio, Beijing, China). Cellular proteins were separated by $12 \%$ SDS-PAGE and transferred to polyvinylidene difluoride (PVDF) membranes (Millipore, Billerica, MA, USA). Membranes were then blocked with $5 \%$ skim milk in TBST $(0.05 \%$ Tween-20) for $1 \mathrm{~h}$ and incubation overnight at $4{ }^{\circ} \mathrm{C}$ with the antibodies antiPRRSV Nsp2 (1:1000), anti-TFDP2 (1:500), anti-p-C/ EBP $\beta$ (1:1000), anti-C/EBP $\beta$ (1:1000), anti- $\beta$-actin (1:5000) or anti-FLAG (1:5000). Then the membranes were washed with PBST three times and incubated with HRP-conjugated secondary antibodies (1:5000) for $1 \mathrm{~h}$. Signals were visualized using Pierce ECL Western Blotting Substrate (Thermo Scientific, Wilmington, DE, USA).

\section{Luciferase Assay}

3D4/21 or HEK293T cells were seeded in 24-well plates and transfected with the constructed plasmids [pRL-TK (20 ng), pGL3-Basic or cyclin A/TFDP2 promoter mutant plasmids (200 ng)] using Lipofectamine 3000 reagent (Invitrogen). At $24 \mathrm{~h}$ post-transfection, the cells were infected with or without PRRSV at a multiplicity of infection (MOI) of 0.5 for $24 \mathrm{~h}$. The lysed samples were prepared and analyzed for firefly and Renilla luciferase activities using a dual-luciferase reporter assay system (Yeasen, Shanghai, China) following the manufacturer's instructions.

\section{RNA Interference}

Small interfering RNAs (siRNAs) targeting TFDP2 (siTFDP2) and C/EBP $\beta$ (siC/EBP $\beta$ ) genes or negative control (NC) were designed and synthesized by GenePharma (Shanghai, China) (Table S3). Briefly, 3D4/21 cells were seeded in 12-well plates and transfected with siRNAs at a final concentration of $50 \mathrm{nmol} / \mathrm{L}$ using Lipofectamine 3000 (Invitrogen). Then cells were infected with $0.5 \mathrm{MOI}$ PRRSV for $24 \mathrm{~h}$. The gene expression levels were assessed by real-time RT-qPCR and Western blotting.

\section{Cell Cycle Synchronization}

3D4/21 cells were cultured in RPMI-1640 medium supplemented with $10 \%$ FBS and then cultured for $24 \mathrm{~h}$ in serum-free supplemented medium (cells arrested in the G0/G1 phase). 3D4/21 cells were seeded in 6-well plates until the cell density was about $80 \%$ following treatment with thymidine ( $2 \mathrm{mmol} / \mathrm{L}$, Sigma, St. Louis, MO, USA), nocodazole ( $80 \mathrm{ng} / \mathrm{mL}$, Selleckchem, Houston, TX, USA) for $24 \mathrm{~h}$ (cells arrested in the S, G2/M phase, respectively).

\section{Flow Cytometry Analysis}

For cell cycle analysis, 3D4/21 cells were seeded in 12 well plates and transfected with $1 \mu \mathrm{g}$ Flag-TFDP2 or vector, or transfected with siTFDP2 or NC at a final concentration of $50 \mathrm{nmol} / \mathrm{L}$. At $12 \mathrm{~h}$ post-transfection, the cells were infected with PRRSV at 0.5 MOI. The cells were harvested and fixed with precooled $70 \%$ ethanol at $4{ }^{\circ} \mathrm{C}$ overnight. Then the cells were incubated with propidium iodide (PI) in the dark for $30 \mathrm{~min}$. The DNA content was analyzed by flow cytometry (BD Biosciences, San Jose, CA, USA).

\section{Confocal Immunofluorescence}

3D4/21 cells in different cell cycles were infected with PRRSV at an MOI of 0.5. At $24 \mathrm{~h}$ postinfection, cells were fixed with $4 \%$ paraformaldehyde and permeabilized with phosphate-buffered saline (PBS) containing 0.3\% Triton X-100 for $10 \mathrm{~min}$. Then cells were blocked with 5\% BSAPBS for $30 \mathrm{~min}$ and stained with anti-Nsp2 antibody for $1 \mathrm{~h}$ at room temperature. Following a wash performed with PBS three times, cells were incubated with FITC-conjugated goat anti-rabbit $\operatorname{IgG}$ secondary antibody for $1 \mathrm{~h}$. Nuclei were stained with 4,6-diamidino-2-phenylindole (DAPI) (Molecular Probes, Waltham, MA, USA). Immunofluorescence was observed with an Olympus confocal microscope. Images were taken at $\times 100$ magnification.

\section{Determination of Virus Titer}

The virus titer was determined using the $50 \%$ tissue culture infective dose $\left(\mathrm{TCID}_{50}\right)$ method as previously described (Zhang et al. 2018).

\section{Statistical Analysis}

All experiments were performed with at least three independent replicates. Data were analyzed by GraphPad Prism software (version 8.0) and differences were analyzed using Student's $t$-test. A $P$ value $<0.05$ was considered statistically significant. 


\section{A}

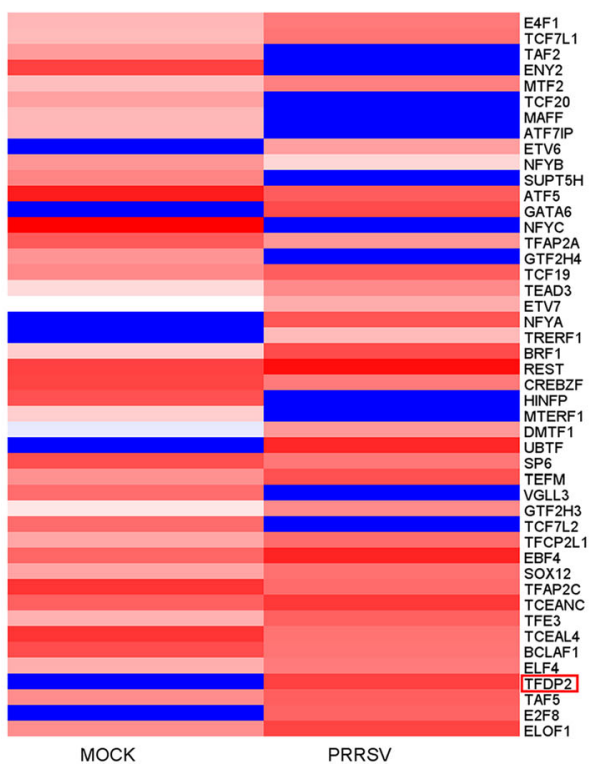

B

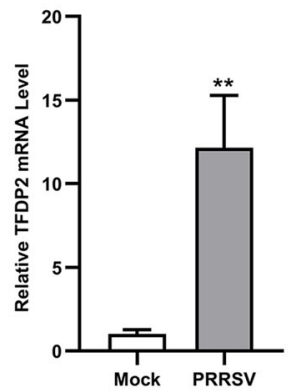

C

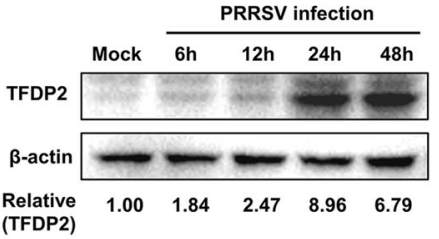

Fig. 1 TFDP2 is upregulated in 3D4/21 by PRRSV. A The heatmap of transcription factors-related differentially expressed genes. B Realtime RT-qPCR analysis of TFDP2 in 3D4/21 cells infected without or with 0.5 MOI PRRSV for $24 \mathrm{~h}$. C 3D4/21 were mock-infected or infected with 0.5 MOI PRRSV. At the indicated time points, cells
A

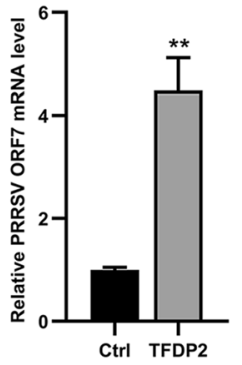

D

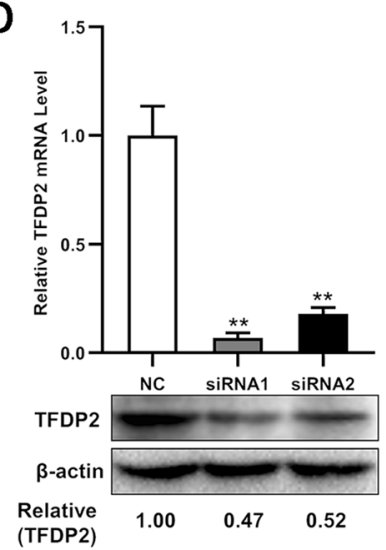

B

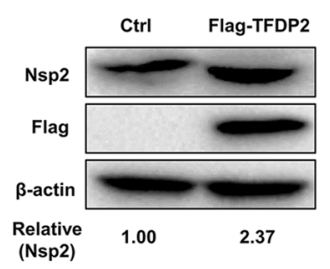

E

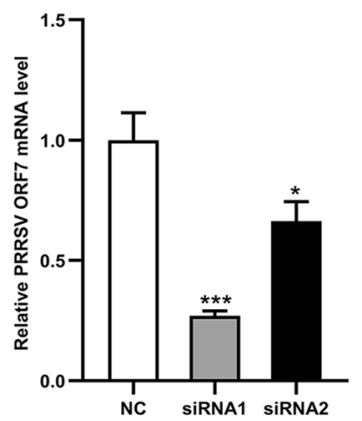

were harvested and subjected to Western blotting (WB) to analyze the expression of TFDP2. Relative expression levels shown below the images were evaluated as fold changes after normalization to $\beta$-actin levels. Data are representative of three independent experiments. Significant differences are indicated as follows: **, $P<0.01$.
C

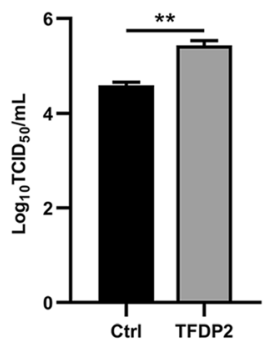

$\mathbf{F}$

G
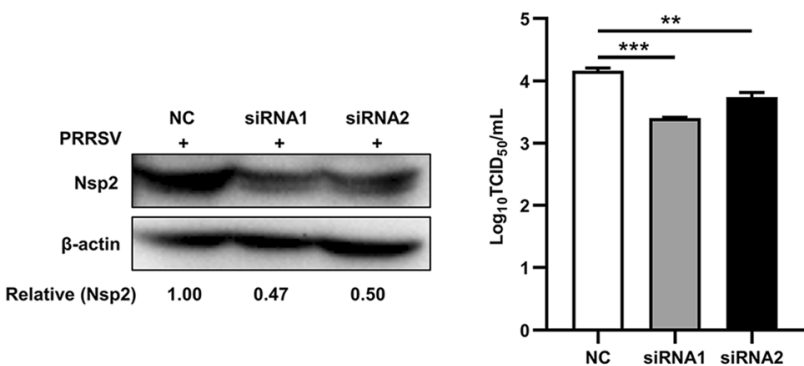

qPCR and Western blot (D). PRRSV ORF7 was detected by real-time RT-qPCR (E), and PRRSV Nsp2 levels were tested by Western blot (F). PRRSV titers in supernatants were tested by $\mathrm{TCID}_{50}$ assay $(\mathbf{G})$. Data are representative of three independent experiments. Significant differences are indicated as follows: *, $P<0.05$; **, $P<0.01$; ***, $P<0.001$. 


\section{Results}

\section{PRRSV Up-Regulates TFDP2 Levels in Viral Infected 3D4/21 Cells}

To screen for genes whose transcription was significantly changed in PRRSV-infected 3D4/21 cells, RNA high throughput sequencing (RNA-Seq) was performed. The mRNA expression profiles of transcription factors showed an up-regulation of TFDP2 after PRRSV infection (Fig. 1A). To further validate the results of the RNA-Seq data, 3D4/21 cells were infected with 0.5 MOI PRRSV. Then total RNA was extracted and the mRNA level of TFDP2 was detected by real-time RT-qPCR. The results showed that PRRSV infection remarkably induced TFDP2 mRNA expression (Fig. 1B). Consistently, Western blot analysis also showed the increased protein level of TFDP2 after PRRSV infection (Fig. 1C). Together, these data demonstrate that PRRSV infection upregulates TFDP2 production in 3D4/21 cells.

\section{TFDP2 Contributes to PRRSV Proliferation}

To figure out if the expression of TFDP2 affects PRRSV proliferation, we overexpressed TFDP2 in 3D4/21 cells and then inoculated with PRRSV for $24 \mathrm{~h}$. It was found that overexpression of TFDP2 increased the mRNA level of the PRRSV open reading frame 7 (ORF7) (Fig. 2A). The results of Western blot analysis showed the increased Nsp2 protein level in TFDP2-transfected cells (Fig. 2B). Meanwhile, the virus titer was higher in cells overexpressing TFDP2 than that of control (Fig. 2C).

To further clarify the above results, we designed two small interfering RNA (siRNA) sequences targeted to TFDP2, and the efficiencies of interference were identified by real-time RT-qPCR and Western blot (Fig. 2D). As shown in Fig. 2E-2G, PRRSV proliferation was suppressed when TFDP2 knockdown. Taken together, these data suggest that TFDP2 facilitates PRRSV proliferation.

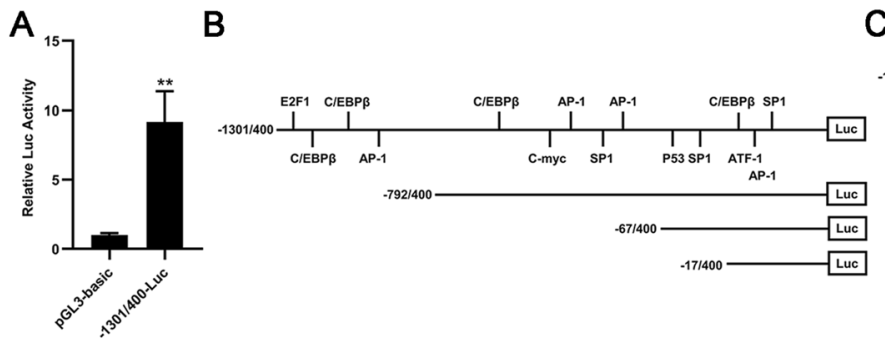

E

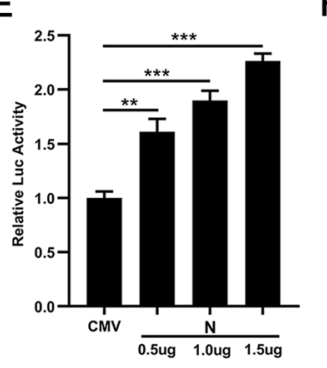

G

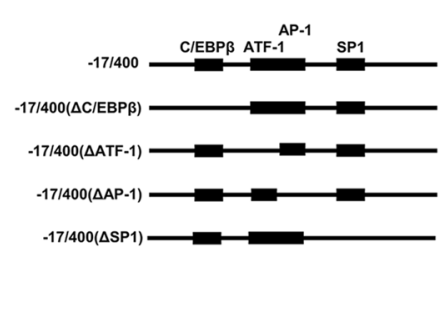

C

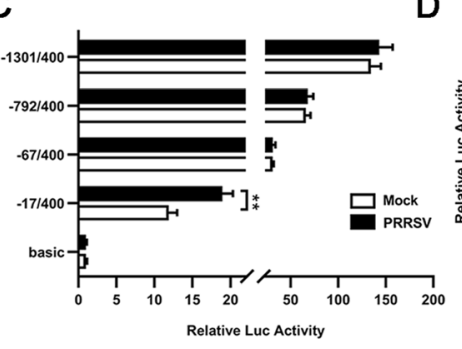

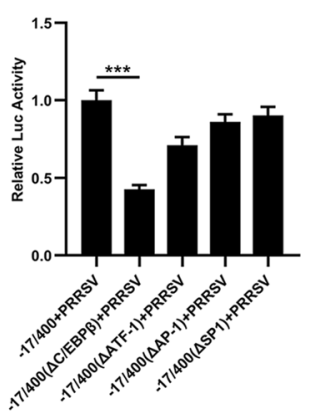

$\mathrm{H}$

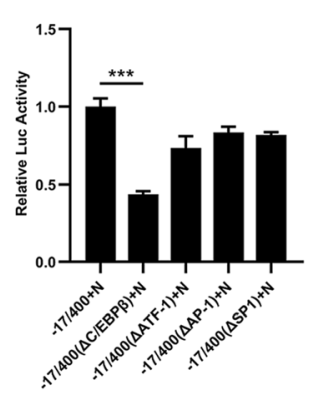

Fig. 3 TFDP2 promoter was activated by PRRSV and its N protein. A, B 1701 bp TFDP2 promoter was cloned into pGL3-basic vector (A). The positions of the putative transcriptional regulatory elements in TFDP2 gene $5^{\prime}$ flanking region were identified and the promoter truncated mutants were generated (B). C The TFDP2 promoter truncated mutants or pGL3-basic and the pRL-TK Renilla luciferase reporter plasmid were co-transfected into 3D4/21 cells. Twenty-four hours later, cells were mock-infected or infected with 0.5 MOI PRRSV. At $24 \mathrm{~h}$ postinfection, cells were collected to determine luciferase activity. D 3D4/21 cells were co-transfected with a series of plasmids that encode PRRSV protein (nsp1 $\alpha$, nsp1 $\beta$, nsp4, nsp5, nsp7, nsp9, nsp10, nsp11, and N) or empty vector, - 17/400-Luc, and the pRL-TK Renilla luciferase reporter plasmid. After $24 \mathrm{~h}$, the cells were harvested for luciferase activity analysis. E 3D4/21 cells were

co-transfected with Flag-N plasmid at doses of $0.5,1.0$, and $1.5 \mu \mathrm{g}$ or empty vector, -17/400-Luc, and the pRL-TK Renilla luciferase reporter plasmid, and the cells were harvested for luciferase activity analysis at $24 \mathrm{~h}$ post-transfection. F Schematic representation of putative TFDP 2 promoter binding sites between -17 to $+400 \mathrm{bp}$ region, including the $\mathrm{C} / \mathrm{EBP} \beta$ ( -11 to -4$)$, ATF-1 $(+30$ to +40$)$, AP-1 (+37 to +43$)$ or SP1 (+79 to +87$)$. $\mathbf{G}$ and $\mathbf{H} 3 \mathrm{D} 4 / 21$ cells were co-transfected with a series of TFDP2 promoter binding sites mutants and the pRL-TK Renilla luciferase reporter plasmid for $24 \mathrm{~h}$ and cells were then infected with 0.5 MOI PRRSV $(\mathbf{G})$ or transfected with Flag-N plasmid $(\mathbf{H})$. Twenty-four hours later, cells were harvested for luciferase activity analysis. Data are representative of three independent experiments. Significant differences are indicated as follows: *, $P<0.05$; **, $P<0.01$; ***, $P<0.001$. 


\section{C/EBP $\beta$ Response Element Is Indispensable for PRRSV and Its N Protein to Activate TFDP2 Promoter}

To investigate the mechanism by which PRRSV induced the transcription of TFDP2, a $1701 \mathrm{bp}$ fragment of the $5^{\prime}$ flanking region of the porcine TFDP2 gene was cloned to construct the TFDP2 promoter-reporter plasmid -1301/ 400 -Luc. And the activity of TFDP 2 promoter was identified by luciferase assay (Fig. 3A). Using the bioinformatics approach (http://alggen.lsi.upc.es) (Bi et al. 2014), we identified several putative transcriptional regulatory elements on the promoter of the TFDP2 gene, and constructed a series of promoter truncated mutants schematically shown in Fig. 3B. To determine the region of the TFDP2 promoter that is responsive to PRRSV infection, 3D4/21 cells were transfected with these constructs. Twenty-four hours after transfection, the cells were infected or mock-infected with 0.5 MOI PRRSV. The luciferase activities of the $-17 / 400$-Luc truncated mutant showed 1.6-fold upregulation upon PRRSV infection, while other mutants did not (Fig. 3C). These results indicate that regulatory elements might exist in the TFDP2 promoter region from -17 to $+400 \mathrm{bp}$ and the responsive elements could be the putative C/EBP $\beta$ ( -11 to -4$)$, ATF-1 $(+30$ to +40$)$, AP-1 $(+37$ to +43$)$ or SP1 $(+79$ to +87$)$. To examine which PRRSV protein is responsible for TFDP2 induction, 3D4/21 cells were co-transfected with the plasmid that encodes PRRSV non-structural and structural protein (nsp1 $\alpha$, nsp1 $\beta$, nsp4, nsp5, nsp7, nsp9, nsp10, nsp11, and N) or empty vector, $-17 / 400$-Luc, and the pRL-TK Renilla luciferase reporter plasmid. The results show that PRRSV $\mathrm{N}$ protein induced the highest TFDP2 promoter activity (Fig. 3D) and in a dose-dependent manner (Fig. 3E).

To further identify the main regulatory element responsive to PRRSV, we mutated putative binding sites of C/EBP $\beta$, ATF-1, AP-1 and SP1 from - 17/400-Luc, respectively (Fig. 3F). The results of luciferase assay demonstrated that mutation in the C/EBP $\beta$ binding site remarkably impaired PRRSV and $\mathrm{N}$-induced activation of TFDP2 promoter (Fig. 3G and 3H). Therefore, these results indicate that the C/EBP $\beta$ binding site of TFDP2 promoter is the main responsive element upon PRRSV infection.

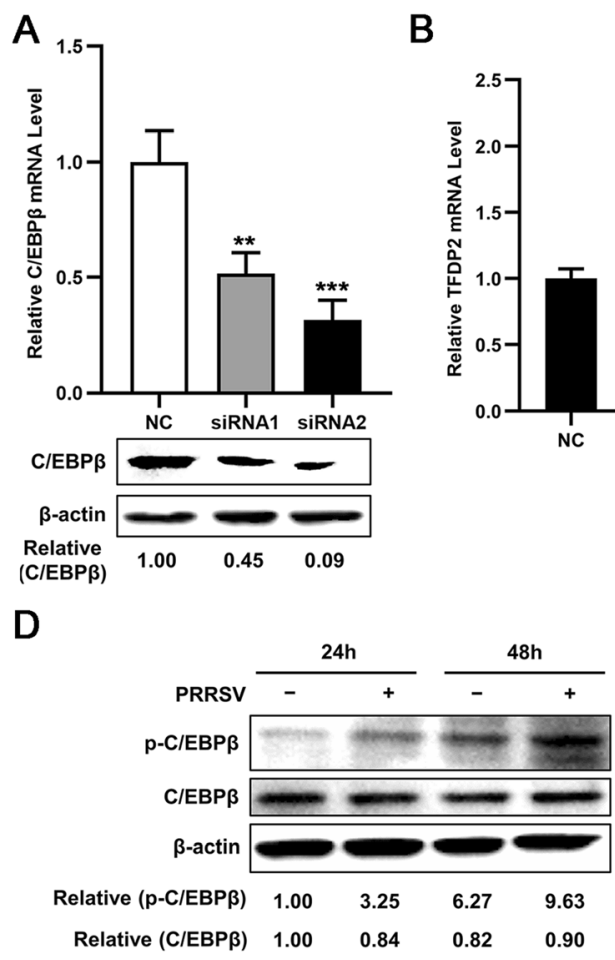

Fig. $4 \mathrm{C} / \mathrm{EBP} \beta$ is required for PRRSV and N-induced TFDP2 expression. A C/EBP $\beta$ siRNAs interference effects were detected by real-time RT-qPCR and Western blot. B, C 3D4/21 cells were transfected with $\mathrm{C} / \mathrm{EBP} \beta$ siRNAs, at $24 \mathrm{~h}$ post-transfection, cells were mock-infected or infected with PRRSV (B), or transfected with Flag-N plasmid or empty vector (C). After $24 \mathrm{~h}$, total RNAs were extracted for TFDP2 mRNA analysis by real-time RT-qPCR. D 3D4/ 21 cells were inoculated with or without PRRSV $(\mathrm{MOI}=0.5$ ) for

E

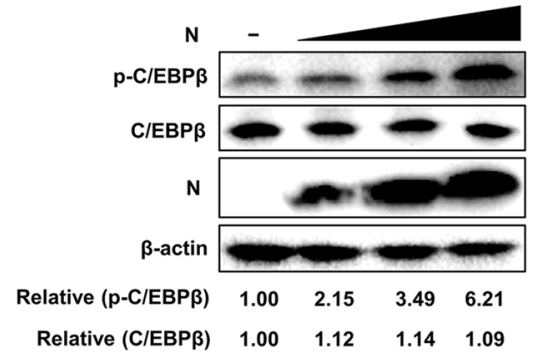

$24 \mathrm{~h}$ or $48 \mathrm{~h}$, and cells were then harvested for $\mathrm{p}-\mathrm{C} / \mathrm{EBP} \beta$ and C/EBP $\beta$ analysis by western blot. E 3D4/21 cells were transfected with Flag-N plasmid at doses of $0.5,1.0$, and $1.5 \mu \mathrm{g}$, an empty vector was used as a control, $24 \mathrm{~h}$ later, cells were collected for analysis of $\mathrm{p}-\mathrm{C} / \mathrm{EBP} \beta$ and $\mathrm{C} / \mathrm{EBP} \beta$ levels using Western blotting. Data are representative of three independent experiments. Significant differences are indicated as follows: **, $P<0.01$; ***, $P<0.001$ 


\section{C/EBPß Is Critical for PRRSV and N-Induced TFDP2 Expression}

To further clarify the role of C/EBP $\beta$ in PRRSV and $\mathrm{N}$-induced TFDP2 production, we detected the effect of knockdown of C/EBP $\beta$ on the TFDP2 expression induced by PRRSV and $\mathrm{N}$ protein using real-time RT-qPCR. The knockdown efficiencies of C/EBP $\beta$ siRNAs were identified by real-time RT-qPCR and Western blot (Fig. 4A). As is shown in Fig. 4B and 4C, silencing C/EBP $\beta$ significantly dampened PRRSV and N-induced up-regulation of TFDP2 expression. These results suggest the involvement of $\mathrm{C} / \mathrm{EBP} \beta$ in PRRSV and N-induced TFDP2 expression.

Previous studies have shown that PRRSV can activate $\mathrm{C} / \mathrm{EBP} \beta$ by enhancing phosphorylation of $\mathrm{C} / \mathrm{EBP} \beta$ ( $\mathrm{Bi}$ et al. 2014; Wang et al. 2019). To validate this finding, 3D4/21 cells were infected with or without PRRSV and harvested at 24 or $48 \mathrm{~h}$ postinfection (hpi), and the level of $\mathrm{p}-\mathrm{C} / \mathrm{EBP} \beta$ and $\mathrm{C} / \mathrm{EBP} \beta$ were analyzed by Western blot. Consistent with previous studies, our results showed increased phosphorylation of C/EBP $\beta$ after PRRSV infection (Fig. 4D). Furthermore, we found that overexpression of PRRSV $\mathrm{N}$ protein increased $\mathrm{p}-\mathrm{C} / \mathrm{EBP} \beta$ level in a dosedependent manner (Fig. 4E). Collectively, these data demonstrate that both PRRSV and its $\mathrm{N}$ protein induce TFDP2 production by activating C/EBP $\beta$.

\section{PRRSV Regulates Cell Cycle at S Phase through TFDP2-Mediated Up-Regulation of Cyclin A}

It has been reported that cyclin $\mathrm{A}$ is the target gene of transcription factors E2F/DP complex, and it controls the $\mathrm{S}$ phase progression of the cell cycle (Yam et al. 2002). We, therefore, speculated whether TFDP2 affects $\mathrm{S}$ phase progression through regulating cyclin A production. First, we constructed the luciferase reporter plasmid of cyclin A and identified its promoter activity (Fig. 5A). Next, we overexpressed TFDP2 to figure out the effect of TFDP2 on cyclin A promoter. The results showed that TFDP2 overexpression induced the promoter activity of cyclin A (Fig. 5B). Real-time RT-qPCR analysis further confirmed that TFDP2 significantly increased the mRNA level of cyclin A (Fig. 5C). We then measured the cell cycle in TFDP2 overexpressed cells. As shown in Fig. 5D,
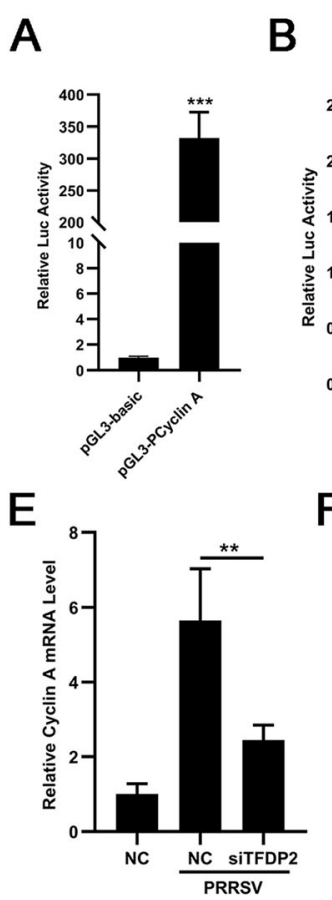

$\mathbf{F}$
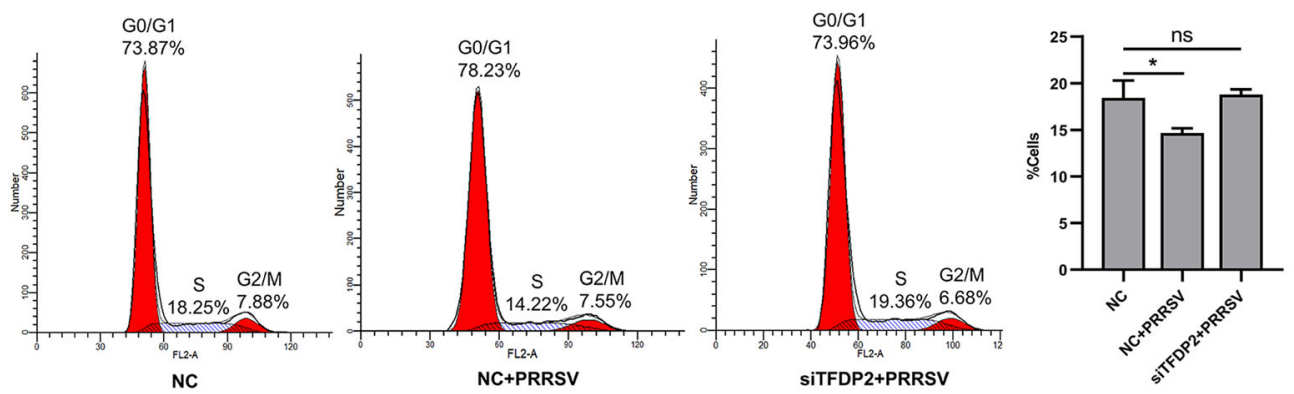

Fig. 5 Effect of TFDP2 on the regulation of the cell cycle in 3D4/21 cells. A Identification of cyclin A promoter activity. 293 T cells were co-transfected with cyclin A promoter-reporter plasmid or pGL3basic and the pRL-TK Renilla luciferase reporter plasmid for $24 \mathrm{~h}$ to detect luciferase activity. B 3D4/21 cells were co-transfected with cyclin A promoter plasmid, the pRL-TK Renilla luciferase reporter plasmid and Flag-TFDP2 or empty vectors. At $24 \mathrm{~h}$ post-transfection, cells were harvested for luciferase activity analysis. C and D 3D4/21 cells were co-transfected Flag-TFDP2 or empty vectors for $24 \mathrm{~h}$, total RNAs were extracted for cyclin A mRNA analysis (C), and cells were stained with propidium iodide and the cell cycle distribution was analyzed by flow cytometry (D). E and F 3D4/21 cells were transfected with NC or siTFDP2 for $24 \mathrm{~h}$ and then mock-infected or infected with 0.5 MOI PRRSV for $24 \mathrm{~h}$. Total RNAs were extracted for cyclin A mRNA analysis (E), and cells were stained with propidium iodide for cell cycle analysis by flow cytometry $(\mathbf{F})$. Data are representative of three independent experiments. Significant differences are indicated as follows: *, $P<0.05$; **, $P<0.01$; ***, $P<0.001 ;$ ns, no significant. 
A

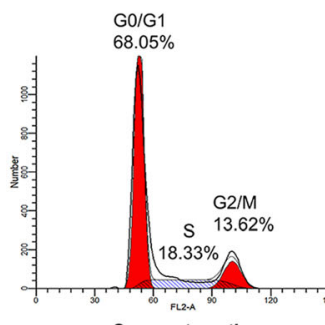

Serum starvation

D

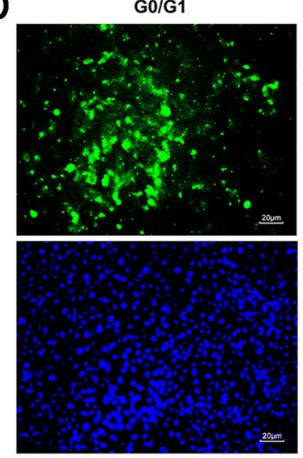

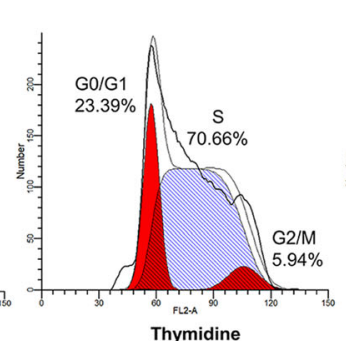

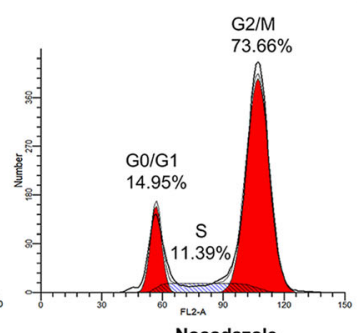

Nocodazole

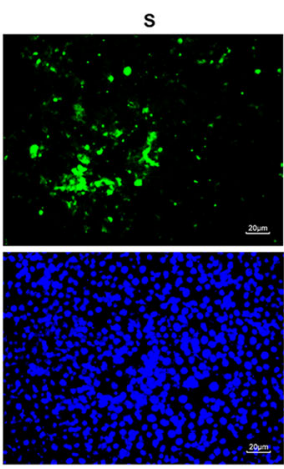

E

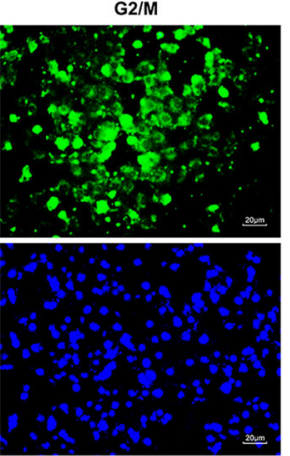

B

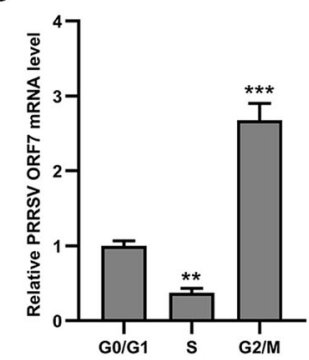

C

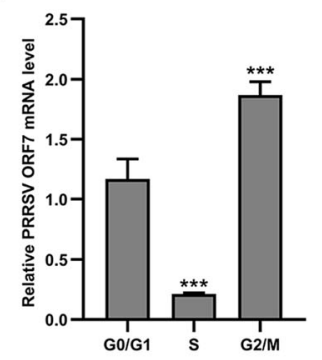

$\mathbf{F}$

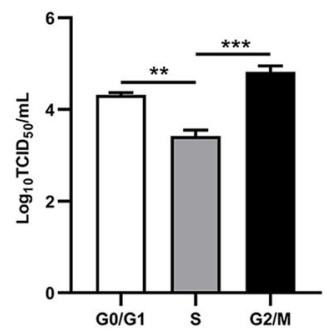

Fig. 6 Sensitivity to PRRSV infection in different cell cycles. A 3D4/ 21 cells were arrested in the G0/G1, S or G2/M phases of the cell cycle using serum starvation, thymidine $(2 \mathrm{mmol} / \mathrm{L})$, or Nocodazole ( $80 \mathrm{ng} / \mathrm{mL})$, respectively. The effect of cell cycle synchronization was examined by flow cytometry. B 3D4/21 cells in different cell cycles were inoculated with PRRSV for $1 \mathrm{~h}$ at $4{ }^{\circ} \mathrm{C}$ and were immediately collected to detect PRRSV ORF7 level by real-time RT-

overexpression of TFDP2 led to a decrease in the number of cells in the $\mathrm{S}$ phase. Meanwhile, we found that PRRSV infection up-regulated the expression of cyclin A, while knockdown of TFDP2 attenuated this up-regulation effect (Fig. 5E). Correspondingly, silencing TFDP2 restored the reduction effect of PRRSV on the number of cells in the $\mathrm{S}$ phase (Fig. 5F). Taken together, these results indicate that PRRSV infection accelerates the $S$ phase progression of the cell cycle through TFDP2-mediated up-regulation of cyclin A expression.

\section{The Decrease of the S Phase Favors the Proliferation of PRRSV}

Accumulating studies have demonstrated that viruses can regulate the host cellular life cycle to favor their proliferation (Fan et al. 2018; Nascimento et al. 2012). To better understand the role of cell cycle on PRRSV proliferation, 3D4/21 cells were synchronized to G0/G1 phase, S phase, or G2/M phase, by cell starvation, thymidine, or nocodazole treatment, respectively. Flow cytometry analysis showed cell cycle stages were arrested successfully by serum starvation and drug treatment (Fig. 6A). First, we examined the relationship between the cell cycle and virus adsorption. PRRSV was inoculated in different cell cycles
qPCR. C-F 3D4/21 cells in different cell cycles were inoculated with 0.5 MOI PRRSV for $24 \mathrm{~h}$. PRRSV ORF7 level was measured by realtime RT-qPCR (C) and PRRSV Nsp2 level was determined by immunofluorescence (scale bar: $20 \mu \mathrm{m})(\mathbf{D})$ and Western blotting $(\mathbf{E})$. PRRSV titers in supernatants were tested by TCID $_{50}$ assay $(\mathbf{F})$. Data are representative of three independent experiments. Significant differences are indicated as follows: **, $P<0.01$; ***, $P<0.001$

for $1 \mathrm{~h}$, after which the cells were immediately collected to detect the PRRSV ORF7 level by real-time RT-qPCR. As shown in Fig. 6B, the cells in the $S$ phase were not conducive to PRRSV adsorption.

To further assess the effects of synchronization at different cell cycle stages on PRRSV replication, PRRSV was inoculated in different cell cycles and the infected cells were harvested after $24 \mathrm{~h}$. Real-time RT-qPCR analysis confirmed that PRRSV ORF7 level in the $\mathrm{S}$ phase was lower than that in the $\mathrm{G} 2 / \mathrm{M}$ or G0/G1 phases (Fig. 6C). Besides, immunofluorescence showed that the expression level of PRRSV Nsp2 was lowest in the S phase (Fig. 6D) and the results of Western blotting further confirmed this finding (Fig. 6E). Furthermore, the virus titers in supernatants of cells in the G2/M or G0/G1 phases appeared greater than that in the $S$ phase (Fig. 6F). The evidence presented indicates that PRRSV replication in 3D4/21 cells is sensitive to the G1/G0 or G2/M phases, rather than the $S$ phase. Together, these results indicate that the cells in the $S$ phase were not conducive to PRRSV adsorption and replication. 


\section{Discussion}

PRRSV is a critical pathogen of swine that brings serious losses to pig farms (Neumann et al. 2005). TFDP2 dimerizes with $\mathrm{E} 2 \mathrm{~F}$ to regulate the transcription of a series of genes, including cell cycle progression, DNA repair, cell differentiation, and apoptosis (Attwooll et al. 2004; DeGregori and Johnson 2006). In this study, we found that the expression of TFDP2 was upregulated during PRRSV infection, and the underlying mechanism was investigated. We constructed the promoter-reporter plasmid of TFDP2 and its truncated mutants. The results of the luciferase assay demonstrated that PRRSV and $\mathrm{N}$ protein activated the promoter activity of TFDP2 to induce its production. We further found that $\mathrm{C} / \mathrm{EBP} \beta$ was essential for PRRSV or $\mathrm{N}$-induced TFDP2 production. The phosphorylation of C/EBP $\beta$ was increased during PRRSV infection and knockdown of C/EBP $\beta$ significantly dampened PRRSV and $\mathrm{N}$-induced expression of TFDP2. It has been reported that PRRSV infection can activate C/EBP $\beta$ (Bi et al. 2014; Wang et al. 2019), which is consistence with our findings.

Next, we investigated the effect of TFDP2 on PRRSV proliferation. Our results showed that overexpression of TFDP2 promoted the proliferation of PRRSV, and, correspondingly, TFDP2 knockdown suppressed PRRSV proliferation. Since TFDP2 is involved in regulating genes about the cell cycle, we speculated that weather PRRSV affected the cell cycle via TFDP2 to promote its proliferation.

Cyclin A plays an important role in controlling the $\mathrm{S}$ phase progression of the cell cycle and its promoter has been shown to be regulated by transcription factors E2F/DP (Yam et al. 2002). Chronic hepatitis C virus (HCV) NS2 protein induces the cell cycle arrest at the $\mathrm{S}$ phase through the down-regulation of cyclin A expression (Yang et al. 2006). PCV2 replication is both $S$ and G2/M phase-dependent, and PCV2 infection down-regulates GPNMB expression, leading to the down-regulation of cyclin $\mathrm{A}$ and the $\mathrm{S}$ phase accumulation, which provides favorable conditions for efficient viral replication (Guo et al. 2019; Tang et al. 2013; Xu et al. 2016). In this study, we found that PRRSV infection decreased the number of cells in the $S$ phase through TFDP2-induced upregulation of cyclin A expression.

PRRSV N protein has been suggested to regulate the cell cycle progression (Yoo et al. 2003). In contrast to our findings that PRRSV infection accelerates the $S$ phase progression, another study demonstrated that PRRSV nsp11 causes the delay of MARC-145 cells at the S phase (Sun et al. 2014). Besides, it has been shown that PRRSV and its truncated glycoprotein $5\left(\mathrm{GP}^{\Delta 97-119}\right)$ can induce arrest of MARC-145 cells at the G2/M phase (Mu et al.
2015; Song et al. 2018), which were not observed in our study. These discrepancies may be due to the use of different cell lines or other experimental conditions. We speculate that the effects of PRRSV infection on the cell cycle may be cell type-specific.

It has been generally accepted that many viruses manipulate the cell cycle of infected cells to regulate and amplify their infection. Rotavirus induction of cell cycle arrest in the $\mathrm{S} / \mathrm{G} 2$ phase enhances viral replication (Glück et al. 2017). Herpesviruses (Flemington 2001), severe acute respiratory syndrome coronavirus (SARS-CoV) $3 \mathrm{~b}$ protein (Yuan et al. 2005), influenza A virus and its NS1 protein (He et al. 2010; Jiang et al. 2013) and murine norovirus (MNV) (Davies et al. 2015) can induce cell cycle arrest in the G0/G1 phase. Enterovirus 71 (EV71) (Yu et al. 2015) and Porcine epidemic diarrhea virus (PEDV) M protein (Xu et al. 2015) mediates cell cycle arrest in the $\mathrm{S}$ phase. We found that synchronization cells in the G0/G1 or G2/M phase rather than the $\mathrm{S}$ phase are more favorable to PRRSV proliferation.

Thus, we believe that PRRSV infection decreases the number of cells in the $S$ phase is beneficial for its proliferation. One possibility is that the $\mathrm{S}$ phase may provide fewer ribonucleotides for PRRSV RNA synthesis than the G0/G1 phase. Since ribonucleotides are precursors of deoxyribonucleotides, and cells in the $\mathrm{S}$ phase will reduce the levels of ribonucleotides (Chen and Makino 2004). Another possibility is that the G2/M phase helps to establish a pseudo-S phase state, which may be more conducive to virus replication (Davy and Doorbar 2007).

In summary, our results showed that PRRSV infection upregulated TFDP2 production in 3D4/21cells by activating transcription factor $\mathrm{C} / \mathrm{EBP} \beta$. Furthermore, our study demonstrated that PRRSV affected the cell cycle progression by regulating TFDP 2 to favor its proliferation. However, the specific molecular mechanism still needs further investigation. In general, this study identified a novel mechanism by which PRRSV utilizes host proteins to promote its infection by affecting the cell cycle.

Acknowledgements This work was supported by the National Key Research and Development Program of China (2018YFD0500500) and the National Natural Science Foundation of China (31272540).

Author Contributions JH conceived and designed the experiments. MZ, XL, RS, PS, AC, LZ performed the experiments. MZ, XL analyzed the data. $\mathrm{MZ}$ and $\mathrm{JH}$ wrote the paper.

\section{Compliance with Ethical Standards}

Conflict of Interest The authors declare that they have no conflict of interest. 
Animal and Human Rights Statement This article does not contain any studies with human or animal subjects performed by any of the authors.

\section{References}

Adams MJ, Lefkowitz EJ, King AM, Harrach B, Harrison RL, Knowles NJ, Kropinski AM, Krupovic M, Kuhn JH, Mushegian AR (2017) Changes to taxonomy and the international code of virus classification and nomenclature ratified by the international committee on taxonomy of viruses (2017). Arch Virol 162:2505-2538

Attwooll C, Denchi EL, Helin K (2004) The E2F family: specific functions and overlapping interests. EMBO J 23:4709-4716

Balistreri G, Viiliäinen J, Turunen M, Diaz R, Lyly L, Pekkonen P, Rantala J, Ojala K, Sarek G, Teesalu M (2016) Oncogenic herpesvirus utilizes stress-induced cell cycle checkpoints for efficient lytic replication. PLoS Path 12:e1005424

Bi Y, Guo X-k, Zhao H, Gao L, Wang L, Tang J, Feng WH (2014) Highly pathogenic porcine reproductive and respiratory syndrome virus induces prostaglandin E2 production through cyclooxygenase 1, which is dependent on the ERK1/2-pC/ EBP- $\beta$ pathway. J Virol 88:2810-2820

Cavanagh D (1997) Nidovirales: a new order comprising Coronaviridae and Arteriviridae. Arch Virol 142:629-633

Chand RJ, Trible BR, Rowland RR (2012) Pathogenesis of porcine reproductive and respiratory syndrome virus. Curr Opin Virol 2:256-263

Chen CJ, Makino S (2004) Murine coronavirus replication induces cell cycle arrest in G0/G1 phase. J Virol 78:5658-5669

Davies C, Brown CM, Westphal D, Ward JM, Ward VK (2015) Murine norovirus replication induces G0/G1 cell cycle arrest in asynchronously growing cells. J Virol 89:6057-6066

Davy C, Doorbar J (2007) G2/M cell cycle arrest in the life cycle of viruses. Virology 368:219-226

DeGregori J, Johnson DG (2006) Distinct and overlapping roles for E2F family members in transcription, proliferation and apoptosis. Curr Mol Med 6:739-748

Dokland T (2010) The structural biology of PRRSV. Virus Res 154:86-97

Fan Y, Sanyal S, Bruzzone R (2018) Breaking bad: how viruses subvert the cell cycle. Front Cell Infect Microbiol 8:396

Flemington EK (2001) Herpesvirus lytic replication and the cell cycle: arresting new developments. J Virol 75:4475-4481

Garner M, Whan I, Gard G, Phillips D (2001) The expected economic impact of selected exotic diseases on the pig industry of Australia. Rev Sci Tech 20:671-686

Glück S, Buttafuoco A, Meier AF, Arnoldi F, Vogt B, Schraner EM, Ackermann M, Eichwald C (2017) Rotavirus replication is correlated with $\mathrm{S} / \mathrm{G} 2$ interphase arrest of the host cell cycle. PLoS ONE 12:e0179607

Grey F, Tirabassi R, Meyers H, Wu G, McWeeney S, Hook L, Nelson JA (2010) A viral microRNA down-regulates multiple cell cycle genes through mRNA 5' UTRs. PLoS Pathog 6:e1000967

Guo K, Xu L, Wu M, Hou Y, Jiang Y, Lv J, Xu P, Fan Z, Zhang R, Xing F (2019) A Host Factor GPNMB Restricts Porcine Circovirus Type 2 (PCV2) Replication and Interacts With PCV2 ORF5 Protein. Front Microbiol 9:3295

He Y, Xu K, Keiner B, Zhou J, Czudai V, Li T, Chen Z, Liu J, Klenk HD, Shu YL (2010) Influenza A virus replication induces cell cycle arrest in G0/G1 phase. J Virol 84:12832-12840

Hitchens M, Robbins PD (2003) The role of the transcription factor DP in apoptosis. Apoptosis 8:461-468
Jiang W, Wang Q, Chen S, Gao S, Song L, Liu P, Huang W (2013) Influenza A virus NS1 induces G0/G1 cell cycle arrest by inhibiting the expression and activity of RhoA protein. J Virol 87:3039-3052

Laichalk LL, Thorley-Lawson DA (2005) Terminal differentiation into plasma cells initiates the replicative cycle of Epstein-Barr virus in vivo. J Virol 79:1296-1307

Lam EW, La Thangue NB (1994) DP and E2F proteins: coordinating transcription with cell cycle progression. Curr Opin Cell Biol 6:859-866

Meulenberg JJ (2000) PRRSV, the virus. Vet Res 31:11-21

Montaner-Tarbes S, del Portillo HA, Montoya M, Fraile L (2019) Key gaps in the knowledge of the porcine respiratory reproductive syndrome virus (PRRSV). Front Vet Sci 6:38

Morgan DO (1995) Principles of CDK regulation. Nature 374:131-134

Mu Y, Li L, Zhang B, Huang B, Gao J, Wang X, Wang C, Xiao S, Zhao Q, Sun Y (2015) Glycoprotein 5 of porcine reproductive and respiratory syndrome virus strain SD16 inhibits viral replication and causes G2/M cell cycle arrest, but does not induce cellular apoptosis in Marc-145 cells. Virology 484:136-145

Music N, Gagnon CA (2010) The role of porcine reproductive and respiratory syndrome (PRRS) virus structural and non-structural proteins in virus pathogenesis. Anim Health Res Rev 11:135-163

Nascimento R, Costa H, Parkhouse R (2012) Virus manipulation of cell cycle. Protoplasma 249:519-528

Neumann EJ, Kliebenstein JB, Johnson CD, Mabry JW, Bush EJ, Seitzinger AH, Green AL, Zimmerman JJ (2005) Assessment of the economic impact of porcine reproductive and respiratory syndrome on swine production in the United States. J Am Vet Med Assoc 227:385-392

Rogers KT, Higgins P, Milla MM, Phillips RS, Horowitz JM (1996) DP-2, a heterodimeric partner of E2F: identification and characterization of DP-2 proteins expressed in vivo. Proc Natl Acad Sci U S A 93:7594-7599

Rossow K (1998) Porcine reproductive and respiratory syndrome. Vet Pathol 35:1-20

Song L, Han X, Jia C, Zhang X, Jiao Y, Du T, Xiao S, Hiscox JA, Zhou EM, Mu Y (2018) Porcine reproductive and respiratory syndrome virus inhibits MARC-145 proliferation via inducing apoptosis and $\mathrm{G} 2 / \mathrm{M}$ arrest by activation of $\mathrm{Chk} / \mathrm{Cdc} 25 \mathrm{C}$ and p53/p21 pathway. Virol J 15:169

Su Y, Shi P, Zhang L, Lu D, Zhao C, Li R, Zhang L, Huang J (2018) The superimposed deubiquitination effect of OTULIN and Porcine Reproductive and Respiratory Syndrome Virus (PRRSV) Nsp11 promotes multiplication of PRRSV. J Virol 92:e00175-18

Sun Y, Li D, Giri S, Prasanth SG, Yoo D (2014) Differential host cell gene expression and regulation of cell cycle progression by nonstructural protein 11 of porcine reproductive and respiratory syndrome virus. Biomed Res Int 2014:430508

Swanton C, Jones N (2001) Strategies in subversion: de-regulation of the mammalian cell cycle by viral gene products. Int J Exp Pathol 82:3-13

Tang Q, Li S, Zhang H, Wei Y, Wu H, Liu J, Wang Y, Liu D, Zhang Z, Liu C (2013) Correlation of the cyclin A expression level with porcine circovirus type 2 propagation efficiency. Arch Virol $158: 2553-2560$

Wang H, Du L, Liu F, Wei Z, Gao L, Feng WH (2019) Highly pathogenic porcine reproductive and respiratory syndrome virus induces interleukin-17 production via activation of the IRAK1PI3K-p38MAPK-C/EBPß/CREB pathways. J Virol 93:e0110001119 
Wang G, Yu Y, Cai X, Zhou EM, Zimmerman JJ (2020) Effects of PRRSV infection on the porcine thymus. Trends Microbiol 28:212-223

Wenzel ES, Singh AT (2018) Cell-cycle checkpoints and aneuploidy on the path to cancer. Vivo 32:1-5

Wu C-L, Zukerberg LR, Ngwu C, Harlow E, Lees JA (1995) In vivo association of E2F and DP family proteins. Mol Cell Biol 15:2536-2546

Xu X, Zhang H, Zhang Q, Dong J, Huang Y, Tong D (2015) Porcine epidemic diarrhea virus $\mathrm{M}$ protein blocks cell cycle progression at S-phase and its subcellular localization in the porcine intestinal epithelial cells. Acta Virol 59:265-275

Xu D, Du Q, Han C, Wang Z, Zhang X, Wang T, Zhao X, Huang Y, Tong D (2016) p53 signaling modulation of cell cycle arrest and viral replication in porcine circovirus type 2 infection cells. Vet Res 47:1-11

Yam C, Fung T, Poon R (2002) Cyclin A in cell cycle control and cancer. Cell Mol Life Sci 59:1317-1326

Yang XJ, Liu J, Ye L, Liao QJ, Wu JG, Gao JR, She Y-L, Wu ZH, Ye LB (2006) HCV NS2 protein inhibits cell proliferation and induces cell cycle arrest in the S-phase in mammalian cells through down-regulation of cyclin A expression. Virus Res 121:134-143

Yoo D, Wootton SK, Li G, Song C, Rowland RR (2003) Colocalization and interaction of the porcine arterivirus nucleocapsid protein with the small nucleolar RNA-associated protein fibrillarin. J Virol 77:12173-12183

Yu J, Zhang L, Ren P, Zhong T, Li Z, Wang Z, Li J, Liu X, Zhao K, Zhang W (2015) Enterovirus 71 mediates cell cycle arrest in $S$ phase through non-structural protein 3D. Cell Cycle 14:425-436

Yuan X, Shan Y, Zhao Z, Chen J, Cong Y (2005) G0/G1 arrest and apoptosis induced by SARS-CoV $3 b$ protein in transfected cells. Virol J 2:66

Zhang Y, Chellappan SP (1995) Cloning and characterization of human DP2, a novel dimerization partner of E2F. Oncogene 10:2085-2093

Zhang L, Ren J, Shi P, Lu D, Zhao C, Su Y, Zhang L, Huang J (2018) The immunological regulation roles of porcine $\beta-1,4$ galactosyltransferase V (B4GALT5) in PRRSV infection. Front Cell Infect Microbiol 8:48 